\title{
Effect of MWCNTs Dispersion and Loading on the Rheological and Electrical Properties of MWCNTs/Silicone Composite
}

\author{
Mingkang Tao ${ }^{1,}$, , Minjie Gong, Tongxin Wang, Feng Chen, Xiaoyun Liu ${ }^{2, b}$, Shaoliang Lin, \\ Qixin Zhuang, c \\ ${ }^{1}$ The Key Laboratory of Advanced Polymer Materials of Shanghai, School of Materials Science and \\ Engineering, Shanghai 200237, China \\ ${ }^{2}$ East China University of Science and Technology, Shanghai 200237, China \\ ataomingkang@hotmail.com, bliuxiaoyun@ecust.edu.cn, ${ }^{\text {}}$ qxzhuang@ecust.edu.cn
}

\begin{abstract}
The dispersion of multiwalled carbon nanotubes (MWCNTs) in silicone was significantly affected by the method of mixing. In this study, rheology showed that there was an optimal mixing time to get the MWCNTs disperse well and high loading of MWCNTs made the suspension flow difficult. The stability of MWCNTs/silicone suspension was analyzed to estimate the degree of re-aggregation during the curing process. The dispersion and loading of MWCNTs affected significantly electrical properties of MWCNTs/silicone elastomer. With the extension of time stirred, the dielectric constant of $1 \mathrm{wt} \% \mathrm{MWCNTs} /$ silicone elastomer increased from 17 to 216 while most high dielectric constant composites were only decades in number. The dielectric loss increased exponentially with the loading of MWCNTs, which made the high dielectric constant meaningless.
\end{abstract}

Keywords: Silicone Elastomer, Multiwalled Carbon Nanotubes (MWCNTs), Electrical Property

\section{Introduction}

In 1991, Iijima discovered carbon nanotubes $(\mathrm{CNTs})^{[1]}$. The CNTs possess low mass density, high aspect ratio and degree of graphitization, which make CNTs endowed with excellent mechanical and electrical properties ${ }^{[2,3]}$. These excellent properties make CNTs promising candidates as filler material in the design of new composite systems. However, the aggregation of CNTs hamper significantly the mechanical and electrical properties of nanocomposites $^{[4-6]}$. Therefore, researching effect of MWCNTs dispersion and loading on properties of composites made by MWCNTs has recently aroused more and more interests of scientists ${ }^{[7,8]}$. 
In this publication, we report that dispersion of multi-wall carbon nanotubes (MWCNTs) in silicone, which was significantly affected by the way of mixing through rheology. An optimized process was selected by comparing the samples' modulus and viscosity in an acceptable mixing time. MWCNTs silicone elastomer with different mixing time and different MWCNTs content were prepared to carry out the effect of MWCNTs on electrical properties. The side effect of high MWNCTs loading was estimated to ensure the composite we made were of practical significance.

\section{Experimental}

MWCNTs (purity, $>95 \%$; outer diameter, $8-15 \mathrm{~nm}$; length, $50 \mu \mathrm{m}$ ) was purchased from XF NANO Materials, Nanjing, China. High temperature vulcanized silicone elastomer obtained from Bluestar Silicones were used as polymer matrix.

Since the ultrasonicaton can cause unwanted heat and damage to $\operatorname{MWCNTs}^{[8,9]}$, mechanical mixing seems to be the appropriate way. The rotate speed is determined by the geometric dimensioning of container and agitator blade. Mixing time varied in order to study the effect of MWCNTs' dispersion on rheological and electrical properties of these composites. $1 \mathrm{wt} \%$ MWCNTs suspensions prepared by mechanical stirring for 10 minutes, 2 hours, 4 hours, 8 hours and 24 hours were labeled as suspension M10M, M2H, M4H, $\mathrm{M} 8 \mathrm{H}, \mathrm{M} 24 \mathrm{H}$ respectively. 1 6 $\mathrm{wt} \%$ MWCNTs and $30 \mathrm{~g}$ silicone were mixed at the same criteria except that the stirring time were fixed at 8 hours. Changing MWCNTs content in order to research the effect of MWCNTs loading on rheological and electrical properties of these composites. Suspension M1wt, M2wt, M3wt, M4wt, M5wt and M6wt were shorted for $1 \sim 6 \mathrm{wt} \%$ MWCNTs suspensions prepared by stirring for 8 hours. On the basis of the above process, these suspensions can be curing into elastomer by being vacuumized and heated.

The rheological properties were carried out on a stress-controlled rotational rheometer HAAKE MARS III (Thermo Fisher scientific, America) at $25^{\circ} \mathrm{C}$ using a $35 \mathrm{~mm}$ parallel plate geometry with the stress of $1 \mathrm{~Pa}$.

Dielectric constant and dielectric loss were measured by using a broad band dielectric spectrometer Concept 40 (NOVOCONTROL, Germany) at $1000 \mathrm{~Hz}$ with disk-shaped samples with $20 \mathrm{~mm}$ in diameter and $2 \mathrm{~mm}$ in thickness. 


\section{Result and discussion}
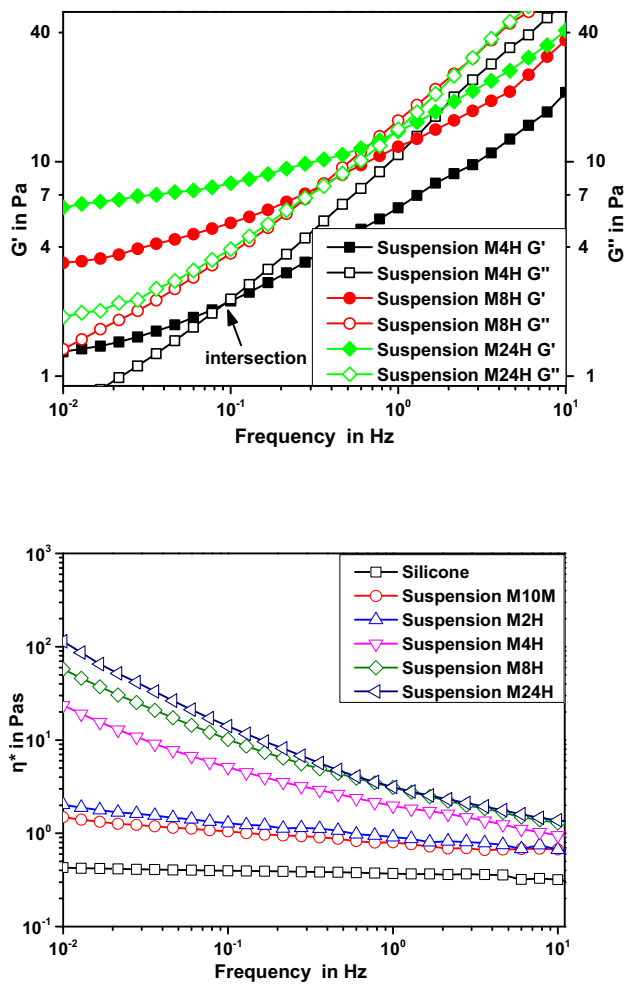

Fig. 1 Modulus and viscosity as functions of frequency for $1 \mathrm{wt} \% \mathrm{MWCNTs} /$ silicone suspensions at different stirring times

Figure 1 shows the curves of modulus and viscosity in function of frequency $(0.01$ $\mathrm{Hz} \sim 10 \mathrm{~Hz}$ ) for the samples with $1 \mathrm{wt} \%$ MWCNTs at $25{ }^{\circ} \mathrm{C}$ and $1 \mathrm{~Pa}$. Both elastic and modulus of MWCNTs/silicone suspensions rose with the increased frequency. Once the dispersion time achieved 4 hours, the elastic modulus and loss modulus intersected at a certain frequency which were called viscoelastic changing point. In the range between 0.01 $\mathrm{Hz}$ and the intersection, the samples exhibited solid-like properties. The viscoelastic changing point moved to higher frequency, when the dispersion time were extended, and the samples exhibited solid-like properties in a greater frequency range.

A shear thinning behavior ${ }^{[8,10]}$ can be observed. The curves of suspension M10M, M2H and pure silicone were substantially parallel, while the viscosity of suspension $\mathrm{M} 4 \mathrm{H}, \mathrm{M} 8 \mathrm{H}$ and $\mathrm{M} 24 \mathrm{H}$ decreased with the increased frequency. The viscosity of suspension prepared by 8 hours stirring increased by 2 orders of magnitude compared to the pure silicone, which illustrated the forming of MWCNTs crosslink network improved modulus and viscosity ${ }^{[11]}$. 
Due to the negligible differences in suspension M8H and suspension M24H, 8 hours should be the optimal stirring time in this paper.
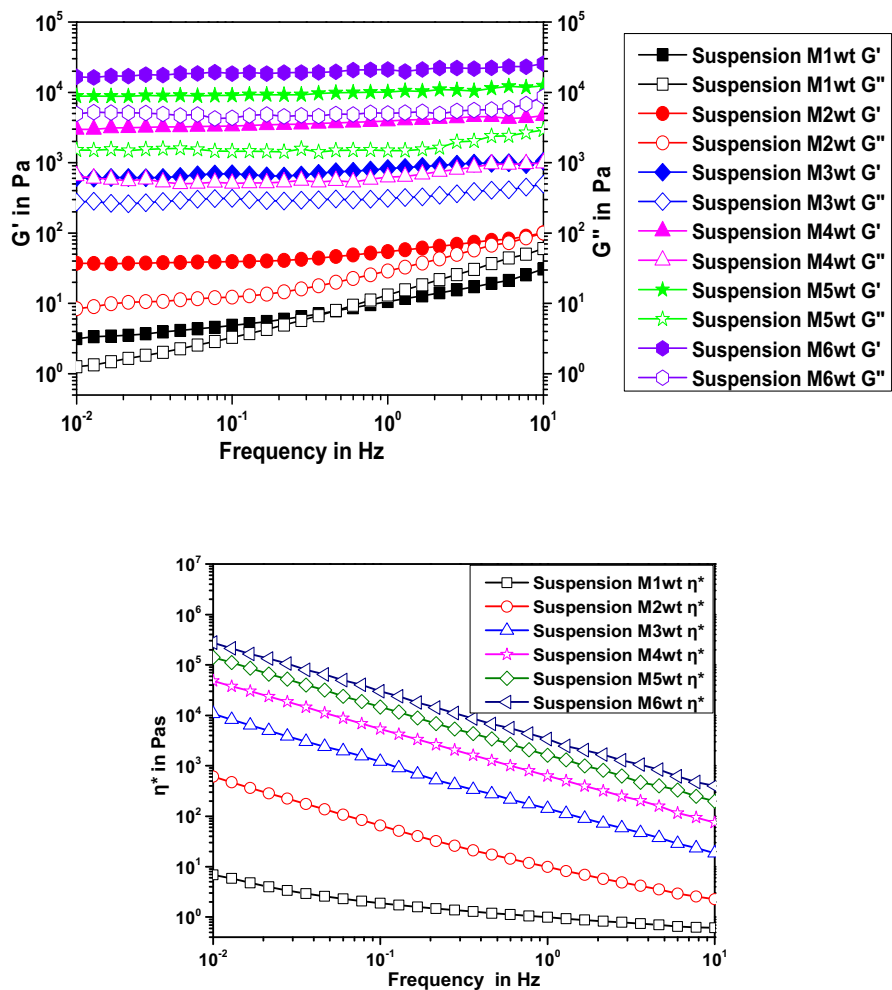

Fig. 2 Modulus and viscosity as functions of frequency for $1 \sim 6 \mathrm{wt} \% \mathrm{MWCNTs} /$ silicone suspensions at 8 hours stirring times

The effect of MWCNTs loading on suspension was also investigated. Once the MWCNTs loading up to $3 \mathrm{wt} \%$, both the elastic modulus and loss modulus were almost frequency independent, as shown in figure 2 . The suspension with $2 \mathrm{wt} \% \mathrm{MWCNT}$ loading could hardly flow anymore, for its vast viscosity. Compared to suspension M24H, the effects of higher MWCNTs loading were significant. The modulus and viscosity of suspension M6wt were almost increased by 5 orders of magnitude compared to the pure silicone, while suspension M24H were only increased by 2 orders of magnitude. Therefore, in order to meet the needs of process and transmission, the amount of carbon nanotubes should be as little as possible in order to keep the viscosity and modulus within a reasonable range. 

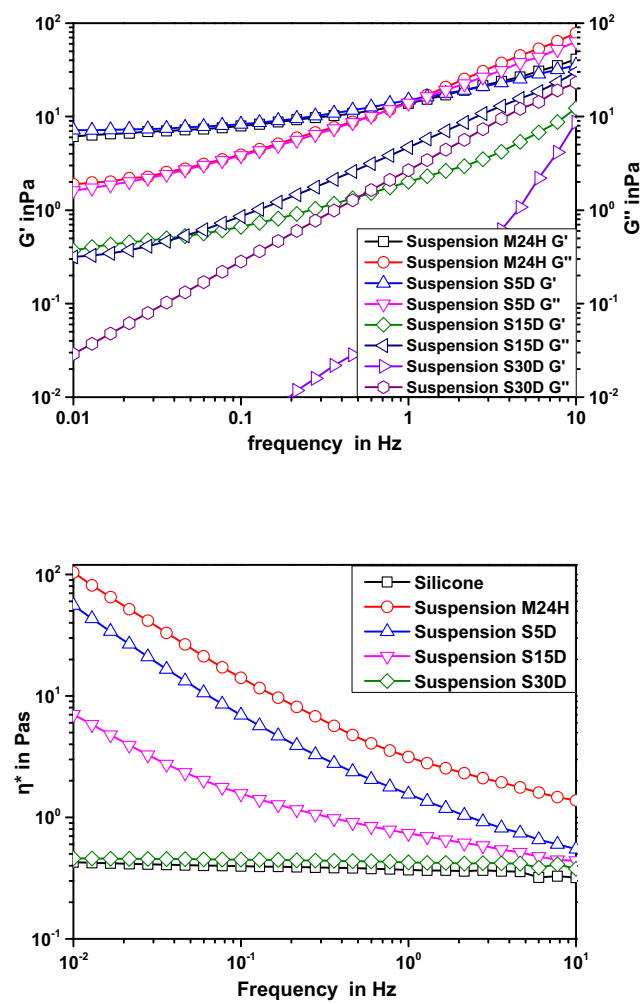

Fig. 3 Modulus and viscosity as functions of suspension M24H placed for different time.

Considering the transformation from suspension to elastomer may take some time, a re-aggregation test should be carried out in order to assess the impact of standing time on it. Suspension S5D, S15D and S30D stand for $1 \mathrm{wt} \%$ MWCNTs suspensions placed for 5 days, 15 days and 30 days. From figure 3, the modulus and viscosity of suspension S5D are very close to suspension $\mathrm{M} 24 \mathrm{H}$, while the suspension placed for over 15 days showed a liquid-like performance. After being stood for a month, the suspension S30D performed like pristine silicone. It may take an hour in the process of curing, which is far less than 5 days. Therefore, a consensus can be carried out that during the curing process, the state of MWCNTs in silicone remains dispersed and re-aggregation does not happen.

In these composite elastomer, well dispersed MWCNTs and their aggregates constitute countless tiny capacitors in the elastomer. Tiny capacitors increased in number and made the dielectric constant greater in number, when MWCNTs dispersed well. 

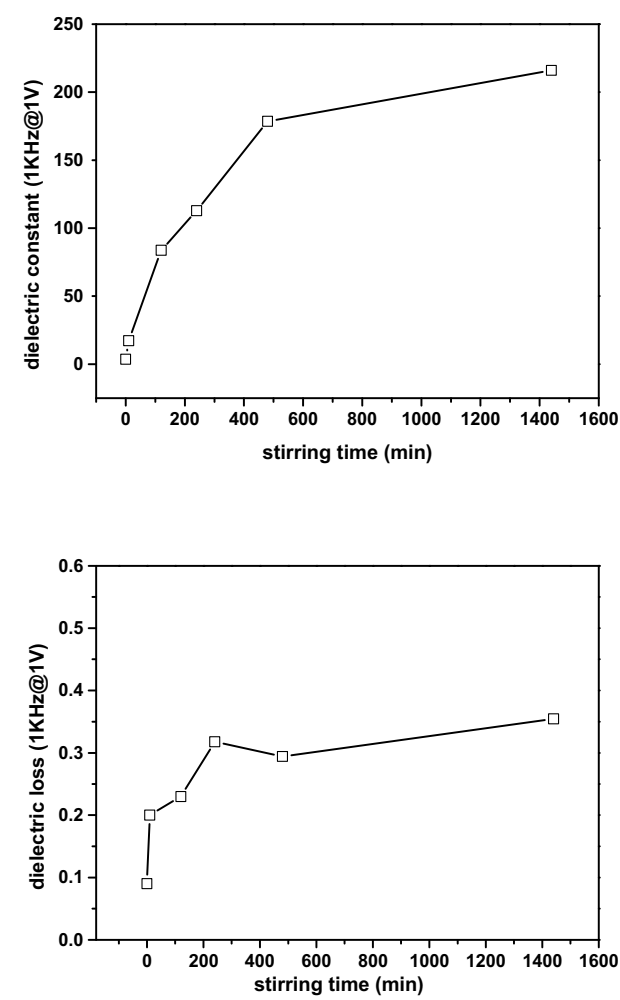

Fig. 4 Dielectric constant and loss as a function of stirring time for $1 \mathrm{wt} \%$

MWCNTs/silicone elastomer

Pristine silicone elastomer owns a quite low dielectric constant, 3 in general, showed in figure 4. The dielectric constant of $1 \mathrm{wt} \%$ MWCNTs elastomer M10M with 10 minutes stirring time was about 20, while the elastomer M24H with 24 hours stirring time was over 200 at $1000 \mathrm{~Hz}$. The results were approving for most composites doped with modified MWCNTs owning a dielectric constant below 70 in number ${ }^{[12,13]}$. Considering the balance of effect between time-cost, 8 hours should be the optimal choice just as the conclusion given by the rheology. The dielectric loss of $1 \mathrm{wt} \%$ elastomer appeared randomness, $0.2 \sim 0.35$, which were quite different from dielectric constant, and seemed to be independent of dispersion time. 

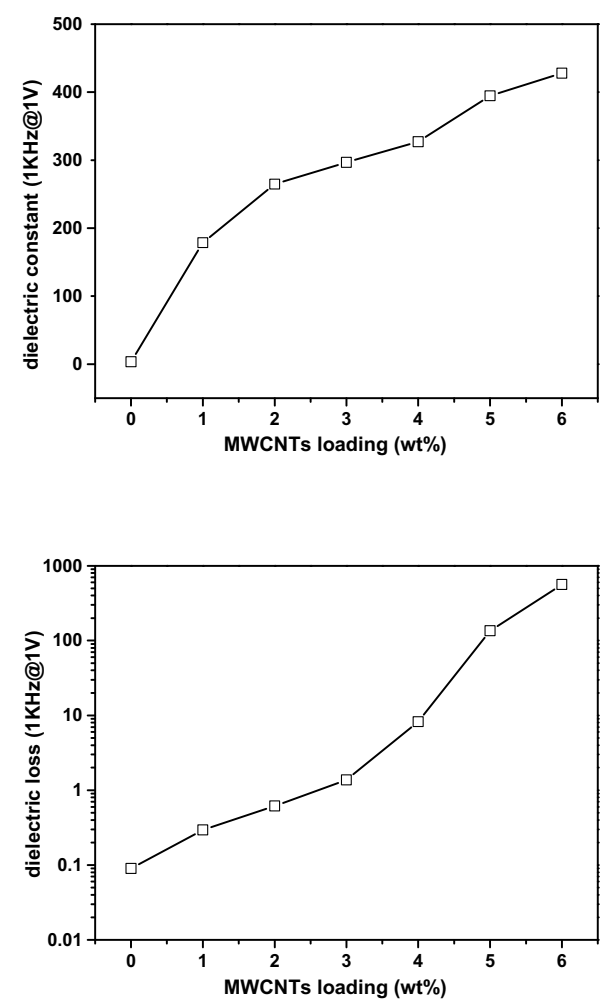

Fig. 5 Dielectric constant and loss as a function of MWCNTs loading for composite elastomer with 8 hours stirring time

The dielectric constant of elastomers increased gradually with the increased MWCNTs loading, while the dielectric losses increased exponentially as shown in figure 5. The dielectric constant of the material reaches 430 at last. When the loading of MWCNTs was up to $3 \mathrm{wt} \%$, and the dielectric loss was over 1 , which means the materials consume more electricity than storing energy. Margin effects are limited, when the content of MWCNTs reaches certain extent, but enormous costs have to be paid. It is not advisable to gain high dielectric constant by increasing MWCNTs loading, because the dielectric loss increased 4 orders of magnitude.

\section{Conclusion}

In the present paper, we investigate systematically how MWCNTs dispersion degree and loading affect both the rheological behavior and electrical properties. For the given mixing method, there is an optimal mixing time to get the MWCNTs dispersed well. 8 hours should be the optimal mixing time in this paper. Once the loading of MWCNTs was over $2 \mathrm{wt} \%$, the suspension became too thick to flow freely. Significant increase of 
dielectric constant was achieved as a result of exceeded mixing time and high loading of MWCNTs. The dielectric constant increased about 2.4 times while the dielectric loss increased 2000 times when the MWCNTs increased from $1 \mathrm{wt} \%$ to $6 \mathrm{wt} \%$. The dielectric loss exponentially increased with the loading of MWCNTs.

\section{Acknowledgements}

This work was financially supported by China National Bluestar(Group) Co. Ltd and the Key Laboratory of Advanced Polymer Materials of Shanghai (ZD20150202).

\section{References}

1. Iijima, S. Helical microtubules of graphitic carbon. Nature 1991, 354, 56-58.

2. Worsley, M. A.; Kucheyev, S. O.; Kuntz, J. D.; Hamza, A. V.; Satcher, J. J. H.; Baumann, T. F. Stiff and electrically conductive composites of carbon nanotube aerogels and polymers. Journal of Materials Chemistry 2009, 19, 3370-3372.

3. Hong, J. S.; Lee, J. H.; Nam, Y. W. Dispersion of solvent-wet carbon nanotubes for electrical CNT/polydimethylsiloxane composite. Carbon 2013, 61, 577-584.

4. Kokott, S.; Heymann, L.; Motz, G. Rheology and processability of multi-walled carbon nanotubes - ABSE polycarbosilazane composites. Journal of the European Ceramic Society 2008, 28, 1015-1021.

5. Nobile, M. R.; Simon, G. P.; Valentino, O.; Morcom, M. Rheological and structure investigation of melt mixed multi-walled carbon nanotube/PE composites. Macromolecular Symposia 2007, 247, 78-87.

6. Berber, S.; Kwon, Y. K.; Tomanek, D. Electronic and structural properties of carbon nanohorns. Phys. Rev. B 2000, 62, R2291-R2294.

7. Quigley, J. P.; Herrington, K.; Baird, D. G. Enhanced electrical properties of polycarbonate/carbon nanotube nanocomposites prepared by a supercritical carbon dioxide aided melt blending method. Polymer 2014, 55, 6167-6175.

8. Huang, Y. Y.; Terentjev, E. M. Dispersion and rheology of carbon nanotubes in polymers. International Journal of Material Forming 2008, 1, 63-74.

9. Lu, K. L.; Lago, R. M.; Chen, Y. K.; Green, M. L. H.; Harris, P. J. F.; Tsang, S. C. Mechanical damage of carbon nanotubes by ultrasound. Carbon 1996, 34, 814-816.

10. Yokozeki, T.; Schulz, S. C.; Buschhorn, S. T.; Schulte, K. Investigation of shear thinning behavior and microstructures of MWCNT/epoxy and CNF/epoxy suspensions under steady shear conditions. Eur. Polym. J. 2012, 48, 1042-1049.

11. Zhang, Q.; Fang, F.; Zhao, X.; Li, Y.; Zhu, M.; Chen, D. Use of dynamic rheological behavior to estimate the dispersion of carbon nanotubes in carbon nanotube/polymer composites. The Journal of Physical Chemistry B 2008, 112, 12606-12611. 
12. Chen, Y.; Lin, B.; Zhang, X.; Wang, J.; Lai, C.; Sun, Y.; Liu, Y.; Yang, H. Enhanced dielectric properties of amino-modified-CNT/polyimide composite films with a sandwich structure. Journal of Materials Chemistry A 2014, 2, 14118-14126.

13. Yang, C.; Lin, Y. H.; Nan, C. W. Modified carbon nanotube composites with high dielectric constant, low dielectric loss and large energy density. Carbon 2009, 47, 1096-1101. 